\title{
Principios de la bioética comprometidos en la detección neonatal
}

\section{Bioethical principles involved in neonatal screening}

\author{
*Ascurra M \\ Programa de Prevención de la Fibrosis Quística y del Retardo Mental. Ministerio de Salud \\ Pública y Bienestar Social (MSP y BS). Asunción, Paraguay
}

\section{RESUMEN}

En este manuscrito se busca identificar los principios de la bioética comprometidos con la detección neonatal, considerando que cualquier acto médico, por más banal que fuese, tendrá sus consecuencias en el ámbito de la ética, especialmente cuando éste es realizado en un recién nacido, que por sus características debe ser considerado de alta vulnerabilidad, involucrando los principios de autonomía, beneficencia y justicia, entre otros. A su vez, por el tenor del tema se abordan principios de la bioética personalista como el derecho a la vida. Estos estudios, caracterizados por su rol en la prevención del daño al recién nacido, también tienen una connotación bioética para las generaciones futuras. Se incluyen el principio de responsabilidad, haciendo referencia a mujeres con fenilcetonuria, donde un embarazo no controlado o irresponsable por parte de la misma, daría lugar al nacimiento de un niño con discapacidad. A los principios mencionados y considerados por su compromiso durante el proceso en sí, existen otros no menos graves con repercusión en cualquier etapa de la vida de los individuos pesquisados, como la estigmatización de personas afectadas pero asintomáticas que podrían ser discriminadas por ello. La información recabada en este manuscrito corresponde a la bioética y la detección neonatal de la literatura nacional e internacional, sistematizada en los principios de bioética, los protagonistas y espacios involucrados en el proceso así como las leyes y garantías jurídicas existentes en el Paraguay. Se concluye en una discusión donde se analizan y entremezclan la bioética y la detección neonatal.

Palabras claves: detección neonatal, principios de bioética.

\section{ABSTRACT}

This manuscript tries to identify the bioethical principles compromised with neonatal detection considering that any medical act, even the most simple one, will have consequences in the ethical field, especially when is carried out in a newborn, who should be seen as a highly vulnerable person because of its characteristics, involving the autonomy, beneficence and justice principles among others. Also, due to the subject other principles of personalist bioethics such as the right to life are approached. These studies, characterized by their role in the prevention of the damage to newborns, have also a bioethical connotation for future generations. The principle of responsibility is also included, making special reference to women with phenylketonuria, taking into account that a non-controlled or irresponsible pregnancy could give birth to a child with a disability. Apart from the principles above mentioned and considered because of their compromise during the process, there are others, which are not less serious, with consequences in any stage of life of the studied individuals, such as the stigmatization of affected but asymptomatic people that could be discriminated. The information contained in this manuscript corresponds to bioethics and neonatal detection collected from national and international literature, systematized in the principles of bioethics, protagonists and spaces involved in the process as well as the laws and legal guarantees existing in

\footnotetext{
*Autor Correspondiente: Dra. Marta Ascurra, Programa de Prevención de la Fibrosis Quística y del Retardo Mental. Ministerio de Salud Pública y Bienestar Social (MSP y BS). Asunción, Paraguay

Email: marta.ascurra@gmail.com

Fecha de recepción: noviembre 2014; Fecha de aceptación: enero 2015
} 
Paraguay. It concludes in a discussion where bioethics and neonatal detection are analyzed and intermingled.

Keywords: neonatal detection, principles of bioethics.

\section{DESARROLLO}

La ética es una reflexión crítica sobre los valores y principios que guían las decisiones y comportamientos humanos. Siendo la bioética un neologismo, acuñado por Van Rensselaer Potter en el año 1971, compuesta de dos términos griegos: Bios que significa vida, concretamente referida a la vida humana, y Ethos que significa costumbre $(1,2)$. Inicialmente considerada como la ciencia de la sobrevivencia humana, nacida con el fin de promover y defender la dignidad humana y la calidad de vida, llegando a abarcar incluso el cosmos y la ecología. Definida como el "Estudio sistemático de la conducta humana en el área de las ciencias de la vida y del cuidado sanitario, en cuanto tal conducta se examina a la luz de los valores y de los principios morales" $(1,3)$.

La ética biomédica, a su vez, puede ser considerada una disciplina especial de la ética, destinada a la resolución de los conflictos y dilemas que la atención en salud plantean a diario y en especial aquellas que surgen con las nuevas tecnologías biomédicas. Tanto la ética biomédica como la bioética se ocupan de los problemas morales del acto médico, entendiéndose este como el conjunto de acciones que recibe el usuario o paciente en los servicios de salud, las cuales realizadas por un profesional de la salud, tienen como objeto el diagnóstico y recuperación del paciente. Si bien éste básicamente es ejercido por el médico, también puede ser realizado por otro profesional de la salud, llámese obstetra, enfermera, etc. (4).

El tamizaje, pesquisa o detección neonatal, screening en inglés, se inicia en los Estados Unidos en la década de 1960, de la mano del Dr. Robert Guthrie, quien descubrió la técnica para el diagnostico neonatal de la fenilcetonuria. Es un acto médico que se realiza en un grupo poblacional especifico (recién nacidos) a fin de identificar a aquellos individuos que presenten un riesgo incrementado de padecer una enfermedad. Una vez hecha la identificación y posterior diagnóstico, debe iniciarse el tratamiento, evitándose así los posibles daños que el individuo en cuestión pueda sufrir de no ser detectado y tratado precozmente. Por lo general, la detección neonatal forma parte del cuadro básico de atenciones que se brinda en salud pública, prevención secundaria. Las patologías incluidas en un programa de detección neonatal, pueden variar de país a país y mismo dentro de una misma población. Esto se debe a que las mismas son elegidas de acuerdo a criterios de selección entre los cuales se destacan que la patología tamizada pueda causar daños graves e irreversibles, carezca de signos clínicos en el periodo neonatal; posea un tratamiento efectivo, de instalación precoz; posea una incidencia suficientemente alta para justificar su investigación; cuente con métodos bioquímicos de diagnóstico y confirmación, sensibles, específicos, sencillos y de bajo costo y tenga un bajo número de falsos positivos y ningún falso negativo. Debe de estar a cargo de una organización estatal y ser aceptada por la comunidad y los profesionales de la salud $(5,6)$.

En Paraguay, la detección neonatal se inicia en 1999, como un programa piloto del Instituto de investigaciones en Ciencias de la Salud, para detectar el hipotiroidismo congénito y la fenilcetonuria en un hospital de la red de salud del Ministerio de Salud Pública y Bienestar Social (MSPyBS). Los resultados obtenidos fueron categóricos, estableciéndose en este estudio una incidencia para el hipotiroidismo congénito de 1 por cada 1.000 a 1.500 recién nacidos (RN). Esto dio pie a la Ley 2.138 en el año 2003, que creó el Programa de Prevención de la Fibrosis Quistica y del Retardo Mental, producidos por el hipotiroidismo congénito y o la fenilcetonuria (7). En el país nacen unos 150.000 recién nacidos al año, de los cuales el $80 \%$, unos 120.000 RN, es atendido dentro de la red de salud del MSPyBS. Este programa se amplía año tras año y actualmente cuenta con más de 150 sitios de toma de muestra distribuidos en los 17 departamentos en los 
que se divide el Paraguay. La implementación del programa ha implicado la capacitación a los profesionales de salud, así como la difusión de este estudio en la comunidad. El programa ofrece el diagnóstico y tratamiento gratuitos de manera masiva para el hipotiroidismo congénito y la fenilcetonuria, no así para la fibrosis quística, que son ofrecidos selectivamente ante la presencia de síntomas en el periodo neonatal 0 antecedentes familiares (8).

A continuación, describimos:

1) Los principios de la bioética que podrían verse involucrados en mayor o menor medida en la detección neonatal;

2) Los principales protagonistas así como los espacios involucrados en la realización del proceso de detección neonatal, incluyendo algunas breves nociones sobre este procedimiento, dirigidas en especial al lector inexperto en el tema y necesarias para la comprensión del trabajo

\section{Principios de la bioética}

La detección neonatal, por el mero hecho de ser un acto médico dirigido a un grupo altamente vulnerable, tiene implicancias muy significativas a nivel bioético que ameritan el conocimiento y una reflexión entre todos los responsables de esta acción. Uno de los modelos de análisis bioético mas difundidos es el principialismo, presentado por Tom Beauchamp y James Childress, en el libro Principios de la ética biomédica, por tanto con raíces en la ética médica $(1,9)$ En él, los autores proponen la existencia de cuatro principios bioéticos (esbozos morales), que regirían un buen manejo ético: no maleficencia y justicia, no dependientes de la voluntad, derivados de los referentes morales ubicados en un nivel superior y por tanto de obligado cumplimiento. Mientras que en un segundo nivel, aquellos que dependen de la voluntad del individuo, la autonomía y la beneficencia. A su vez otros principios como: la confidencialidad, la proporcionalidad, la veracidad, la responsabilidad y otros que a razón de la autora de esta monografía podrían verse envueltos durante todo el proceso que conlleva un estudio de detección neonatal, desde el nacimiento hasta la vida adulta del individuo investigado. Al final se incluye por el tenor del tema algunos principios de la bioética personalista, como el principio de defensa de la vida física, de libertad y responsabilidad, así como el de la totalidad y el de sociabilidad y subsidiaridad.

A continuación se describe el alcance de cada uno de los principios comprometidos con la detección neonatal:

Principios de la bioética principialista

Principio de autonomía: también conocido como de autodeterminación o capacidad para realizar actos con conocimiento de causa y sin coacción $(1,9,10)$. La autonomía personal es reconocida como aquella donde la regulación personal de uno mismo es posible, sin interferencias externas que pretendan controlar, y sin limitaciones personales. Como principio de la ética biomédica, significa que debe respetarse la decisión del paciente, después de una adecuada información referente al o los procedimientos o tratamientos que se podrían aplicar al mismo. Implica por tanto dos elementos fundamentales, el raciocinio o entendimiento necesario para sopesar lo ofertado así como la libertad para aceptar o rechazar. Oponiéndose al paternalismo médico, en presencia de una buena relación médico-paciente. El principio de autonomía se ve pues reflejado en su máxima expresión a través del consentimiento informado $(11,12)$ donde el consentir no es simplemente aceptar someterse a un tratamiento, cualquiera fuese éste, es el enfermo quién asume y toma la decisión de someterse o no a un tratamiento y por ende a sus consecuencias.

Principio de beneficencia: Extremar los posibles beneficios y minimizar los riesgos (1, 2, 10). Este principio consiste en un compromiso activo, hacer el bien, persigue el bien terapéutico del paciente. Es quizás el principio más aplicado de la ética biomédica en la 
relación médico-paciente. De ahí que en algunos casos, prevalezca por sobre el principio de autonomía por lo menos en el modelo paternalista

Principio de No maleficencia: No hacer mal a otra persona (1, 2, 10). Este principio proclama no causar un daño. Todo ser humano tiene la obligación moral de respetar la vida y la integridad física de las personas, aún en el caso que éstas autoricen para actuar en contrario, nadie tiene la obligación de hacer el bien a otro en contra de su voluntad, pero sí está obligado a no hacerle mal. Esta obligación es exigida a todos por igual, incluso en forma coercitiva.

Principio de justicia o equidad: No discriminar a las personas en su vida social $(1,2,10)$. Establece que "todas las personas tienen derecho a igual calidad de salud". Por ello, todos los ciudadanos deben tener acceso a una atención médica de calidad. En el ámbito biomédico, corresponde a la justicia distributiva. La distribución equitativa de los derechos, beneficios y responsabilidades o cargas en la sociedad. Distribución moralmente correcta de las cargas y beneficios en una sociedad.

A los principios inicialmente promulgados se suman otros como:

Principio de confidencialidad: Este principio parte del secreto médico, presente en el Juramento Hipocrático. La excepción corresponde a la posibilidad de ocasionar daños a terceros o a la sociedad. La información por tanto solo debe ser conocida por los involucrados en el acto médico y solo será revelada cuando esta revelación no pueda causar daño a la persona $(1,10,13)$.

Principio de la proporcionalidad: Como en las acciones médicas existen beneficios y riesgos, es indispensable tener junto al principio de beneficencia otro principio, el de proporcionalidad, que en el momento de tomar las decisiones y cuando se ponen en la balanza costos y beneficios, pueda justificar determinados riesgos ante los beneficios aportados (14).

Principios de la bioética personalista:

Estos se basan en la persona humana y su bien. Se analizan aquellos que podrían verse envueltos en un acto médico y correspondan a la dimensión física o espiritual del ser humano. Atendiendo que en esta acción el usuario principal se halla representado por terceros, los cuales a su vez adquieren derechos a ser tenidos en cuenta y por tanto respetados.

El Personalismo, es la doctrina moral del cristianismo que afirma en primer lugar que los valores éticos son universales y fundamentales, mientras que en segundo lugar menciona el principio de santidad de la vida. Siendo Dios el creador de la vida, la misma es sagrada y por tanto ningún ser humano podrá disponer voluntariamente de ella.

Principio de defensa de la vida física: La vida física corporal del hombre representa un valor fundamental a través del cual se manifiesta la persona y expresa valores tales como la libertad y la sociabilidad. El respeto a la vida, así como su defensa y promoción, constituyen el imperativo ético más importante del hombre, en el que se destaca la defensa de su salud (15).

Principio de libertad y responsabilidad: La libertad debe ejercerse responsablemente ante la vida propia y la ajena. La vida y la salud se han encomendado a la responsabilidad del paciente, en el caso de los recién nacidos de sus padres o tutores, con la ayuda de los profesionales de la salud (15).

Principio de totalidad o terapéutico: La corporeidad humana es un todo unitario. Existe una totalidad física, espiritual y moral de la persona. Este principio de totalidad rige la licitud y obligatoriedad de la terapia médica y quirúrgica, de ahí que se lo conoce también como principio terapéutico (15).

Principio de sociabilidad y subsidiaridad: La vida y la salud corresponden a un bien social. Toda persona debe comprometerse a considerar su propia vida y salud y la de los demás como un bien personal y social. Este principio obliga a la comunidad a promover la vida y salud de todos y cada uno. La subsidiaridad comienza por el respeto a la autonomía del 
paciente, es decir, atender a sus necesidades sin sustituirle su capacidad de decidir y actuar (15).

\section{Actores del proceso de detección neonatal}

Primero se describirá a los protagonistas principalmente involucrados en este acto médico, para luego continuar con los espacios donde se lleva a cabo el proceso de detección neonatal. Si bien estos pueden ser divididos en dos grandes grupos: el público y el privado, solo se tendrá en cuenta el escenario o espacio publico, por considerar que esta actividad, por el beneficio que acarrea a la persona afectada, la familia y porque no a la comunidad, no debería ser encarada como una actividad de lucro por las instituciones privadas.

Así entre los protagonistas identificados figuran:

El usuario o paciente: en la detección neonatal, un recién nacido pasivo, incapaz de pensar lo que es bueno para él o de articular algún reclamo y por tanto altamente vulnerable, dependiendo en todos los sentidos de sus representantes y del estado para ejercer sus derechos. El concepto vulnerable se enmarca en la definición clásica de "debilidad, fragilidad" $(10,16)$, en atención a que la vulnerabilidad es inespecífica, ya que todos somos vulnerables a diferentes riesgos y en diferentes situaciones.

Los representantes, padres o tutores del recién nacido: principales responsables de su salud, por tanto forman parte esencial de este grupo de protagonistas. A pesar de ser un acto médico casi podríamos decir inocuo, ya que se infringe un daño insignificante a la hora de la toma de muestra, más aún al sopesar con el beneficio que podría ser obtenido en caso de estar en riesgo de desarrollar una patología. Estos actores adquieren un mayor protagonismo en presencia de un recién nacido en riesgo, teniendo en cuenta que la toma de muestra es solo el inicio de la detección neonatal, que culmina muchas veces con un individuo adulto libre de las complicaciones propias de la patología y por tanto con la capacidad para hacerse cargo de su propia autonomía (10).

Los profesionales de la salud: encargados de brindar la información a los representantes legales del recién nacido sobre la existencia del estudio, de llevar a cabo una suerte de consentimiento informado verbal, para luego proceder al pasaje de datos de la ficha y concluir con la extracción de la toma de muestra. Se suman al listado de los protagonistas, principalmente a la hora de analizar temas que hacen a la bioética, debido a que en el ámbito hospitalario aún hoy conservan un comportamiento paternalista, considerando que la información a los padres o tutores del recién nacido no debe ser entregada, lo cual se refuerza con la presencia de una ley de obligatoriedad del estudio a todos los recién nacidos. Por otro lado, se debe de mencionar también otros puntos en relación a los profesionales de la salud, los cuales en muchos casos, en especial en nuestro país, se hallan saturados de trabajo entre otras situaciones, ubicándolos también en la posición de sujeto vulnerable $(10,17)$.

Las instituciones sanitarias: la detección neonatal si bien puede ser desarrollada en cualquier sitio, es un acto médico, por lo tanto son las instituciones sanitarias, hospitales, centros y puestos de salud del país, donde debe de ser ofrecido este estudio, de preferencia en aquellos donde ocurren nacimientos o acuden los recién nacidos para su atención. Para esto el lugar debe contar con la infraestructura necesaria que permita una atención humanizada y de calidad, siendo el estado el responsable de asistir y cubrir esta atención, a todos los recién nacidos sin ningún tipo de discriminación, compartiendo con los padres o tutores esta responsabilidad (10).

El Programa de Prevención de la Fibrosis Quistica y del Retardo Mental: fue creado por la Ley 2.138 en el 2.003, posteriormente por el Decreto Presidencial No 2126 del 2004, es incorporado al ministerio de Salud Publica y Bienestar Social. El programa ofrece de manera gratuita a todos los recién nacidos del país el estudio del hipotiroidismo congénito (HC) y la fenilcetonuria (PKU, por sus siglas en inglés), de manera selectiva el estudio de la fibrosis quística (FQ), esto porque el análisis de costo: beneficio, no resulta positivo, a 
diferencia del costo: beneficio que implica la atención de las dos patologías primeras. A su vez son ofrecidos los medicamentos, alimentos y análisis para las dos primeras, mientras que para la FQ la atención no es global, por el elevado costo del tratamiento de cada individuo.

Las muestras enviadas por los más de 150 sitios de toma de muestra distribuidos en las 18 Regiones Sanitarias en las que se divide el país, son ingresadas a una base de datos y codificadas, posteriormente son analizadas en el laboratorio del programa. Tanto las fichas con los datos de los recién nacidos estudiados como las muestras remanentes luego de su estudio, son guardadas en un depósito, para su destino final al cabo de cinco o más años $(6,7)$.

Se discute y analiza la relación del acto médico de la detección neonatal con la bioética desde el punto de vista de los principios bioéticos principialistas, personalistas y legales. A fin sistematizar la información, trataremos de ir enlazando de manera ordenada los principios, los actores y las leyes comprometidas con la detección neonatal.

La bioética encarada en un inicio como la ética de la supervivencia y más tarde como la encargada de regir la tecnociencia, constituye ahora la ética aplicada del día a día, a las acciones que de una u otra forma pueden ir contra la dignidad de la persona humana. De ahí que el acto médico de la detección neonatal pueda ser pasible de ser examinado en mayor o menor medida bajo los principios de la bioética principialista y la personalista.

El principio de autonomía o de autodeterminación en el recién nacido, por su incapacidad, no puede serle reconocido, lo que quizás le otorgue una mayor vulnerabilidad. Este concepto merece un párrafo aparte en un trabajo de bioética y detección neonatal, ya que si bien esta palabra hace referencia a una debilidad, fragilidad $(10,16)$, no debemos olvidar que todos somos vulnerables en diferentes ocasiones o ante diferentes riesgos. Con la diferencia que en el recién nacido su vulnerabilidad es protegida por sus padres o tutores encargados de la patria potestad del mismo, pudiendo o no ser ejercido por éstos (18).

En Paraguay, la ley 2138 ha implementado estudios de detección neonatal que permiten detectar en el recién nacido patologías que podrían afectar su salud de no ser tratadas a tiempo. Por ello el artículo 4, obliga todas las instituciones de salud, tanto públicas como privadas del país, donde se producen nacimientos, a realizar los estudios correspondientes a todos los recién nacidos $(7,10-12)$ y a través del articulo 5, responsabiliza a los padres, tutores o guardadores a concurrir dentro de los siete días del nacimiento a un centro asistencial, a los efectos de proceder a la toma de muestras para la realización de los estudios.

Así pues, encontrar un equilibrio entre el derecho a la intimidad que asiste a una familia y la defensa del niño recién nacido contra toda forma de descuido o trato negligente mientras se encuentre bajo la custodia de los padres o de un representante legal que lo tenga a su cargo, no es una tarea sencilla. El Estado delega en las familias el cuidado de su prole y al mismo tiempo, debe asumir el compromiso de apoyarlas a través de las leyes y los recursos económicos para que puedan desempeñar la tarea de manera idónea. La responsabilidad (principio de libertad y responsabilidad de la ética personalista) por la crianza es, por tanto, compartida por la familia y el Estado, con miras a la realización completa del pequeño y por ende, al pleno disfrute de sus derechos. Cuando el núcleo familiar no cumple plenamente con lo pactado y expone al niño a una situación de desatención o negligencia, el Estado, a través de sus medios legales, tiene no sólo el derecho sino también el deber ineludible de inmiscuirse en la vida familiar para proteger a los miembros más indefensos de la comunidad invocando principios ético-sociales de justicia y equidad (10).

Siguiendo con el principio de autonomía, en la detección neonatal éste es respetado por los profesionales a través del consentimiento informado, el cual solo se cumple cuando el encargado de la toma de muestra brinda la información adecuada sobre el propósito del estudio y las implicancias de los resultados, lo que se ha dado en llamar "medicina 
centrada en el paciente" (1). A pesar que el paternalismo, entendido éste como la práctica donde el profesional de la salud, con el fin de beneficiar a una persona, tiende a imponer decisiones al paciente, basado en la teoría que "el profesional es el que sabe". En el caso de la detección neonatal, si la información no es brindada de manera adecuada y adaptada a las posibilidades de comprensión de los padres, contemplando las implicancias personales y socio-familiares, el estudio podría no concretarse debido a que en la época ideal para hacer el estudio el recién nacido no es visto por ninguno de los actores como un paciente $(10-12)$.

El principio de autonomía en la detección neonatal también se enfrenta a la obligatoriedad del estudio de manera legal, lo que permite que en muchos casos, éste no sea considerado y por ende respetado. Podemos también mencionar el principio de protección descrito por Schramm y Kottow, que justifica el no reconocimiento de la autonomía ante los intereses del bien común o como en el caso especifico, del recién nacido $(10,14)$.

En el raro caso de conflicto de intereses con los representantes del recién nacido, los profesionales de la salud, ante la no aceptación de un estudio de detección neonatal, evocando el mejor interés del recién nacido, podrán no respetar esta decisión extrayendo la muestra del recién nacido a través de la intervención judicial. En caso de que el profesional decidiese respetar esta posición y no tuviese el valor de recurrir a los estrados legales para hacer cumplir la obligatoriedad de la ley, debe resguardarse haciendo firmar a los representantes una hoja de rechazo del estudio, solicitando a los mismos un control de salud cercano $(6,18)$.

El principio de beneficencia ha sido en cierta forma abordado, al mencionar que la detección neonatal permite la prevención del retardo mental o una mejor calidad de vida de los afectados por las patologías pesquisadas. Este beneficio es innegable si es entregado con el tratamiento adecuado, donde padres o tutores, unidos al Estado como ente encargado de otorgar el presupuesto para hacer efectivo esto, cumplen a cabalidad los roles que les corresponden $(6,7,10)$.

El principio de autonomía prácticamente agotado en el párrafo anterior, no constituye un principio ético absoluto pues admite excepciones, en cuanto es enfrentado al principio de la beneficencia. Por ejemplo, cuanto mayor sea el beneficio para el recién nacido o para la sociedad, la exigencia de someterse o acatar es más obligatoria (10).

En cuanto al Principio de Justicia, ya en el Preámbulo de la Declaración Universal de Derechos Humanos de 10 de diciembre de 1948, se proclaman los principios o valores que impregnan a todo el texto articulado: "la libertad, la justicia y la paz en el mundo tienen por base el reconocimiento de la dignidad intrínseca y de los derechos iguales e inalienables de todos los miembros de la familia humana" siendo el estado, a través de sus organizaciones, el encargado de proteger la vida y garantizar la salud. La detección neonatal, además al ser un tema de salud colectiva, se convierte en un problema de salud pública, pasando así un tema entendido como de ética biomédica a una bioética global al incorporar la justicia distributiva que podría convertirse en una injusticia social si la cobertura no alcanza a los sectores más desprotegidos. Sumándose a éste el principio de sociabilidad-subsidiaridad que obliga a la comunidad a ayudar donde la necesidad sea mayor $(18,19,25)$.

El principio de justicia también incluye al financiamiento continuo, no pudiendo detenerse y reiniciarse un programa dependiendo de los recursos financieros disponibles, pues ello anularía cualquier beneficio posible, sin mencionar la perdida económica que implica la organización y puesta en marcha de este tipo de programa (10). A su vez, el principio de justicia hace hincapié en el hecho de que todas las personas tengan acceso a una asistencia sanitaria digna e igual, teniendo como único criterio prioritario a los más vulnerables y no a los más demandadores. La salud tiene un precio cuando los recursos son limitados pero lo que se puede ofrecer no. El principio de justicia regla en la distribución (10). 
La confidencialidad en relación a los resultados es evidente, el reconocimiento de que no puede ser proporcionada la información a ninguna otra instancia es evidente, en caso de que esto no ocurra puede considerarse un atentado contra la libertad individual $(10,13)$.

Se considera que la muestra de sangre pertenece al individuo y solo puede ser utilizada previo consentimiento informado. Por tanto, no seria ético ni legal la realización de estudios diferentes a los informados, en muestras remanentes de la detección neonatal. En caso en que se desee utilizarla se puede hacerlo ya sea contactando con las familias de los recién nacidos, a fin de conseguir el permiso correspondiente lo cual puede resultar hasta casi imposible o utilizando códigos que no permitan llegar a la identidad de la persona $(13,24)$.

Un principio si bien no muy comprometido con la detección neonatal en sí, es el principio de defensa de la vida física, relacionado en cuanto que una vez que se tenga el diagnóstico en el primer hijo, las familias podrían atentar contra este principio a fin de tener descendencia sin la patología, en caso que el feto esté de nuevo afectado $(5,13$, 15). Esto es posible evitar, informando adecuadamente en especial, sobre el tratamiento de las patologías, lo que permite una buena calidad de vida a los niños detectados y tratados precozmente. A esto podemos agregar, la posibilidad de estigmatización de los individuos detectados, aunque no presenten síntomas de la enfermedad. Ambas situaciones podrían verse beneficiadas con amplias campañas de difusión sobre el tema a la población general, así como la inclusión a nivel de pre y postgrado de los avances en relación al tratamiento de las patologías involucradas.

\section{CONCLUSIÓN}

Fueron identificados diez principios involucrados con la detección neonatal, de los cuales cuatro corresponden a los de la bioética principialista: Principio de autonomía, Principio de beneficencia, Principio de No maleficencia y el Principio de justicia o equidad. Así como otros dos asociados a los primeros: Principio de confidencialidad y Principio de la proporcionalidad. Los otros cuatro restantes, correspondieron a los principios de la bioética personalista: Principio de defensa de la vida física, Principio de libertad y responsabilidad, Principio de totalidad o terapéutico $y$ Principio de sociabilidad y subsidiaridad.

A su vez, fueron reconocidos como actores de la detección neonatal, el recién nacido, identificado como usuario o paciente; los representantes, padres o tutores del recién nacido y los profesionales de la salud. Mientras que el proceso en si tiene como espacio a las instituciones sanitarias, principalmente de la red de salud del Ministerio de Salud Pùblica y Bienestar Social y a la sede del Programa de Prevención de la Fibrosis Quistica y del Retardo Mental

En Paraguay, si bien la detección neonatal se halla legislada siendo obligatoria para todos los recién nacidos, los principios de justicia y equidad aún no han sido cumplidos a cabalidad, por lo cual un alto porcentaje de la población, en especial la más vulnerable, no puede disfrutar del preciado derecho, de ser sano. La patología sigue su curso y es diagnosticada en muchos casos con consecuencias irreversibles, ante un cuadro de retardo mental e incluso la muerte del afectado. La necesidad de fortalecer acciones de prevención como la detección neonatal constituye uno de los retos del sector salud, que se plantea a los planificadores de la misma, con el imperativo de desarrollar un modelo basado en el costo, la eficiencia y la calidad. La revisión y posterior enfoque de las políticas para ofrecer servicios enfocados a las necesidades epidemiológicas y de prevención, con alto impacto económico y social, como es la detección neonatal, constituye un imperativo ético, para mejorar la calidad de vida de la población. 


\section{REFERENCIAS BIBLIOGRÁFICAS}

1. Mestral E d, Lugo E, Martínez de Nuñez I, Rivarola J, Mestral de Gimenez E de, Samaniego C, Ayala de Mendoza F, Mazcotti U, Bianco H. Manual de bioética. Asunción: EFACIM; 2006.

2. Pessini L, Bachifontaine CP. Problemas atuais de bioética. 7a ed. San Paulo: Ediciones Loyola; 2005.

3. Enciclopedia de bioética, 2da edición. Vol 1. W.T. Reich, editor responsable, 1995.

4. Perales Cabrera A. El acto médico: Critérios, definición y límites. Diagnostico /Internet/. 2001 /citado 10 jul 2014); 40(1). Disponible en:

http://www.fihu-diagnostico.org.pe/revista/numeros/2001/enefeb01/46-52.html

5. Colombo M, Cornejo V, Raimann E. Errores innatos en el metabolismo del niño. $2^{a}$ ed. Chile: Editorial Universitaria; 2003.

6. Ministério de Salud Publica y Bienestar Social. Guia de toma de muestra del programa de prevenciòn de fibrosis quistica y retardo mental. Asunción: Ministério de Salud Publica y Bienestar Social; 2009.

7. Prevenciòn de la fibrosis quistica y del retardo mental. Ley 2138 (13 jun 2003).

8. Ascurra M, Rodríguez S, Valenzuela A, Blanco F, Ortiz L, Samudio M. Incidencia de hipotiroidismo congénito en catorce regiones sanitarias del Paraguay. Pediatr (Asunción). 2009; 36(2). 111-5.

9. Beauchamp TL, Childress J. Principios de ética biomédica. Barcelona: Masson; 1999.

10. Tealdi JC, director Diccionario latinoamericano de bioética. Bogotá: UNESCO; 2008.

11. Cecchetto S. Teoría y practica del consentimiento informado en el área neonatal. Bueno Aires:

Ediciones Suárez. Serie Filosofía; 2000.

12. Junges JR. Exigencias ética do consentimento informado. Revista bioética. 2007; 15(1): 77-82.

13. UNESCO. Declaración universal sobre bioética y derechos humanos. Pamplona: UNESCO; 2005

14. Kottow M. Salud pública, genética y ética. Rev Saúde Pública 2002; 36(5): 537-44.

15. Burgos JM. El personalismo: Temas y autores de una filosofía nueva. 2a ed. Madrid: Palabra; 2004.

16. Stalsett SJ. Vulnerabilidad, dignidad y justicia: valores éticos fundamentales en un mundo globalizado En: Encuentro internacional: Las dimensiones eticas del desarrollo /Internet/. 2003: 3 y 4 jul; Belo Horizonte; 2003. /citado 10 jul 2014/. Disponible en:

http://www.hacienda.go.cr/centro/datos/Articulo/Vulnerabilidad,\%20dignidad\%20y\%20justicia.pdf

17. Abdalla-Filho E. Violencia em saúde: Quando o médico e o vulnéravel. Bioética. 2004; 12(2):121-6.

18. Comisión Nacional Constituyente. Constitución nacional del Paraguay 1992, Asunciòn. (20 de junio, 1992).

19. Asamblea General de las Naciones Unidas. Declaración universal de los derechos humanos, Resolución 217. (10 de diciembre, 1948).

20. Convención Americana sobre Derechos Humanos suscrita en la Conferencia especializada Interamericana sobre Derechos Humanos. San José, Costa Rica. (7 al 22 de noviembre, 1969).

21. Naciones Unidad Derechos Humanos. Pacto internacional de derechos económicos, sociales y culturales. Adoptado y abierto a la firma, ratificación y adhesión por la Asamblea General en su resolución 2200 A (XXI), (16 de diciembre, 1966).

22. Fondo de las Naciones Unidas para la Infanc- UNICEF. Convención internacional sobre los derechos del niño y de la niña. Asunciòn: UNICEF; 2007.

23. Declaración Americana de los Derechos y Deberes del Hombre. Aprobada en la Novena Conferencia Internacional Americana Bogotá, Colombia. (1948).

24. Declaración Universal sobre el Genoma Humano y los Derechos Humanos. (11 de noviembre, 1997).

25. Lantos JD, Meadow WL. Bioética neonatal: Los desafíos morales de la innovación médica. Baltimore: Imprenta Universidad John Hopkins; 2006. 